\title{
Tackling metastasis
}

\author{
A deeper understanding of the molecular and cellular underpinnings of metastatic disease and a renewed focus on \\ metastasis-targeting therapeutic approaches raise hopes for improved clinical translation.
}

Ti: hat metastasis is the leading cause of cancer-related death sadly remains a truism in the cancer field. Despite the fact that outcomes for patients with cancer have improved dramatically over the past few decades, with cancer-related mortality in the United States alone dropping by $29 \%$ since the early 1990 s, survival rates for patients with metastatic disease remain dismal ${ }^{1}$. A prime reason for the limited clinical progress is that metastasis is a systemic, multi-step process, with context-dependent features ascribed to each particular tumor type, the colonized organ and its microenvironment.

Layered over this complexity is the lengthy time to the development of detectable metastatic disease, which has proven a formidable foe in the efforts to tackle metastasis. Few of the many cancer cells that leave the primary tumor will eventually initiate metastatic outgrowth in distal sites, and the manifestation of metastasis typically occurs after long periods - on the order of several months to years - of undetectable disease after treatment of the primary tumor. A major factor in this is tumor dormancy: the ability of disseminated tumor cells (DTCs) to enter a prolonged state of growth arrest until conditions governed by their microenvironment reactivate them to fuel metastatic outgrowth ${ }^{2}$. Understanding the biology that underlies the initiation of and awakening from dormancy is key in the efforts to improve outcomes for patients with cancer.

Building on the literature that identified the extracellular matrix (ECM) as an important microenvironmental component of the metastatic niche, in this issue of Nature Cancer, Di Martino et al. study the interplay between DTCs and their surrounding ECM in controlling tumor dormancy ${ }^{3}$. Using established head-and-neck and breast cancer cell line models of dormancy and sophisticated imaging methods, the authors define the collagen architecture of dormant tumor nodules and individual cells versus that of their proliferating counterparts, and observe that remodeling of collagen fibers to a more linear orientation is linked to the reactivation of DTCs from dormancy. Defining the matrisome of dormant cancer cells versus that of proliferative cancer cells and characterizing its functional importance in mouse tumor-implantation experiments in vivo, they show that dormant cancer cells produce an ECM rich in type III collagen, which endows such microenvironments with a wavy ECM organization and the ability to induce and maintain dormancy. They further demonstrate that expression of type III collagen and establishment of this dormancy phenotype requires signaling through the collagen receptor DDR1 and transcription factor STAT1. Although more work is needed to pave a route to clinical translation, these findings raise the intriguing possibility of detecting metastatic recurrence through altered ECM architecture and of using type III collagen and ECM remodeling therapeutically to suppress metastatic tumor outgrowth by reinforcing a dormant state on tumor cells.

Highlighting the functional complexity of the ECM microenvironment, a conceptually related study by Sun et al., published in Nature, demonstrates that DDR1-dependent collagen fiber alignment leads to the exclusion of immune cells from tumors ${ }^{4}$. This work shows that targeting this mechanism with DDR1-neutralizing antibodies can reduce the growth of established tumors in mice. In a Nature Cancer News \& Views article, Birgit Leitinger discusses the implications of these differing DDR1-related effects for cancer therapy ${ }^{5}$.

In a separate Nature Cancer article, Dai et al. address the effects of the ECM on tumor dormancy in a specialized microenvironment - that of the brain ${ }^{6}$. They show that breast cancer cells disseminate to the mouse brain and occupy the perivascular niche there, with those that remain dormant being located at specialized structures of astrocytes called 'endfeet'. At these locations, astrocyte-mediated production of the laminin-211 ECM protein induces DTC quiescence by binding dystroglycan on the surface of DTCs. This specific ECM interaction subsequently leads to cytoplasmic sequestration of the transcriptional co-activator YAP and the rewiring of tumor-cell gene-expression programs to promote dormancy. These findings provide valuable insight into the metastatic process in the hard-to-treat environment of the brain. In an accompanying News \& Views article, Khan and Steeg offer their perspective on how this work advances the understanding of dormancy and brain metastasis? .

Switching gears from tumor dormancy to metastatic growth, two Nature Cancer studies by Kang and colleagues explore the anti-metastatic effects of pharmacologically targeting the interaction between metadherin (MTDH) and staphylococcal nuclease domain-containing 1 (SND1) $)^{8,9}$, a protein complex that is known to be involved in tumor initiation, metastasis and therapy resistance. In their first study, the authors characterize the pro-metastatic role of the MTDH-SND1 complex in more detail through the use of an inducible $M t d h$-knockout mouse to demonstrate that loss of this interaction inhibits mammary tumor progression and metastasis ${ }^{8}$. Notably, they go on to identify a class of small chemical inhibitors that disrupt this protein-protein interaction in breast cancer cells and demonstrate that these compounds can inhibit tumor growth and metastasis and can sensitize tumor cells to chemotherapy.

In the companion study, Kang and colleagues use these tools to explore the systemic underpinnings of the pro-metastatic roles of MTDH and SND $1^{9}$. They find that this protein complex enhances metastasis of mammary tumors by binding to the mRNAs encoding the Tap 1 and Tap 2 components of the antigen-presentation machinery and promoting their degradation, thereby inhibiting the presentation of tumor antigens. This prevents $\mathrm{T}$ cell activation, which allows metastatic tumor cells to evade clearance by $\mathrm{CD} 8^{+}$cytotoxic T cells. Conversely, pharmacological inhibition of the MTDH-SND1 complex counteracts immunoevasion by promoting $\mathrm{T}$ cell activation, which the authors show can synergize with anti-PD1 immunotherapy to reduce metastasis in mice harboring metastatic breast cancer cells.

In an accompanying News \& Views article, Jiang and Ganesh discuss the potential of these inhibitors of the MTDH-SND1 interaction and their combinatorial effects with chemotherapy and immunotherapy in treating metastatic breast cancer ${ }^{10}$.

The complexity of the metastatic process has for a long time been prohibitive for the development of efficacious treatments, and it is improbable that single-agent 
approaches will offer a broad therapeutic solution. Although much more work is needed to achieve clinical translation, the studies presented in this issue raise the promise of future treatment solutions by providing a deeper understanding of tumor dormancy and the systemic and microenvironmental signals that influence the biology of metastasis, and by identifying pharmacological means to modulate metastasis in synergy with other therapeutic agents.

Published online: 20 January 2022

https://doi.org/10.1038/s43018-021-00327-0

References

1. Esposito, M., Ganesan, S. \& Kang, Y. Nat. Cancer 2, 258-270 (2021).

2. Risson, E. et al. Nat. Cancer 1, 672-680 (2020).

3. Di Martino, J.S. et al. Nat. Cancer https://doi.org/10.1038/s43018 021-00291-9 (2021).
4. Sun, X. et al. Nature 599, 673-678 (2021)

5. Leitinger, B. Nat. Cancer https://doi.org/10.1038/s43018-02100323-4 (2022).

6. Dai, J. et al. Nat. Cancer https://doi.org/10.1038/s43018-02100297-3 (2021)

7. Khan, I. \& Steeg, P. S. Nat. Cancer https://doi.org/10.1038/s43018021-00321-6 (2021)

8. Shen, M. et al. Nat. Cancer https://doi.org/10.1038/s43018-02100279-5 (2021).

9. Shen, M. et al. Nat. Cancer https://doi.org/10.1038/s43018-02100280-y (2021).

10. Jiang, Q. \& Ganesh, K. Nat. Cancer https://doi.org/10.1038/ s43018-021-00320-7 (2022). 